\title{
Achievements and Limits on the Controlled Release of a Drug from a Textile Fabric to Dermis
}

\author{
Radu Cezar-Doru ${ }^{1, *}$, Popa Marcel $^{1}$, Parteni Oana $^{1}$, Salariu Mihaela ${ }^{1}$, Lupușoru Elena-Cătălina ${ }^{2}$, \\ Ghiciuc Cristina $^{2}$, Foia Liliana ${ }^{2}$, Chiriac Anca ${ }^{2}$, Lupusoru Raoul ${ }^{2}$, Oproiu Loti ${ }^{3}$ and Ulea Eugen ${ }^{4}$ \\ ${ }^{I}$ Technical University Iasi, street Mangeron 29, Romania \\ ${ }^{2}$ University of Medicine and Pharmacy Iaşi, Romania \\ ${ }^{3}$ ICECHIM Institute Bucharest, Romania \\ ${ }^{4}$ University of Agricultural Sciences and Veterinary Medicine Iasi, Romania
}

\begin{abstract}
The paper presents the overall results of theoretical achievements and experimental details when designing a material for a textile fabric, which releases a drug for a specific pathology. It presents the conclusions of the researches on the development of textile support as an anti-allergic, anti-fungal and anti-psoriasis type, but also on toxicological, biocompatibility and therapeutic issues for establishing the amount of drug needed for the trans-dermal diffusion. This paper estimates the possibility of applying a cyclodextrin (Cyc) on a textile surface to form temporary reservoirs by complexing and subsequently releasing the drug under the action of cutaneous stimuli. One refers to the achievements of authors, and works submitted by other research groups in the area of textile substrates used as implant or underwear worn next to the skin. The results are analyzed both as a scientific communication and the possible application for a potential current industrial processing.
\end{abstract}

Keywords: Cutaneous pathologies, cyclodextrin, drug release, hydrogels, matrices, textile fabric.

\section{INTRODUCTION}

The fibrous polymers form textiles of external medical use (fasciae, bandages, patches), for protection equipment (coats, bonnets, gloves, trousers), as well as for prosthetic implants (eventration nets, cardiac stents, vascular prostheses, stents). A development potential also presents the textiles worn in direct contact with epidermis, on which systems for controlled release of a drug temporary deposited on the textile surface are applied.

Textile costumes for therapies of cutaneous pathologies were designed and manufactured; the active principle being trans-dermal diffusion from microparticles under the action of stimuli (of enzyme, friction, temperature, humidity and perspiration types) to the corneum layer, mezzo- and hypodermis.

The especially high applicative potential resulting from the trans-dermal transfer is also due to the advantage that it avoids the oral or parental administration of drug, being an efficient solution for the therapies specific to chronic pathologies. As cutaneous pathologies that can be approaches, we can quote: contact and atopic dermatitis, of allergic type, mycoses of various locations [1], psoriasis with palm, plantar, scalp or members location [2]. The advantage

*Address correspondence to this author at the Technical University Iasi, street Mangeron 29, Romania; Tel: +40748830254; Fax: +40232230491;

E-mail: rcezar2010@yahoo.com of the textile with drug release as compared to oral administration is that the drug does not pass through the digestive path, where the loss of active substance diminishes the drug concentration by means of the excretory, digestive or circulatory processes; the drug concentration frequently decreases under the therapeutic minimum. On the other side, it avoids the hepatic metabolism, its administration is easier and more attractive for the patient, and there is the possibility to cease the treatment if necessary.

Fig. (1) illustrates the optimum domain $A B C$ of the variation of the sanguine concentration of the drug during its administration.

What concerns the principle, the utilization of textiles containing drugs on their surface is close to the topical administration in the form of active principle included in an unguent or cream, where the applied therapy can present advantages or restrictions. The increase of drug solubility through different methods has the advantage of diffusion, related to the increase of drugs accessibility within the tissue, therefore the advantage of diminishing the doses prescribed by the clinician, decreasing the social cost of therapy and the adverse effects.

The drug transfer through trans-dermal path has its advantages, but the diffusion speed is obstructed by the corneous layer, which represents an effective barrier for the trans-cellular or even intra-cellular diffusion, this limitation depending on the transfer localization on the body surface, and on the diffusion ability due to drug structure. The 


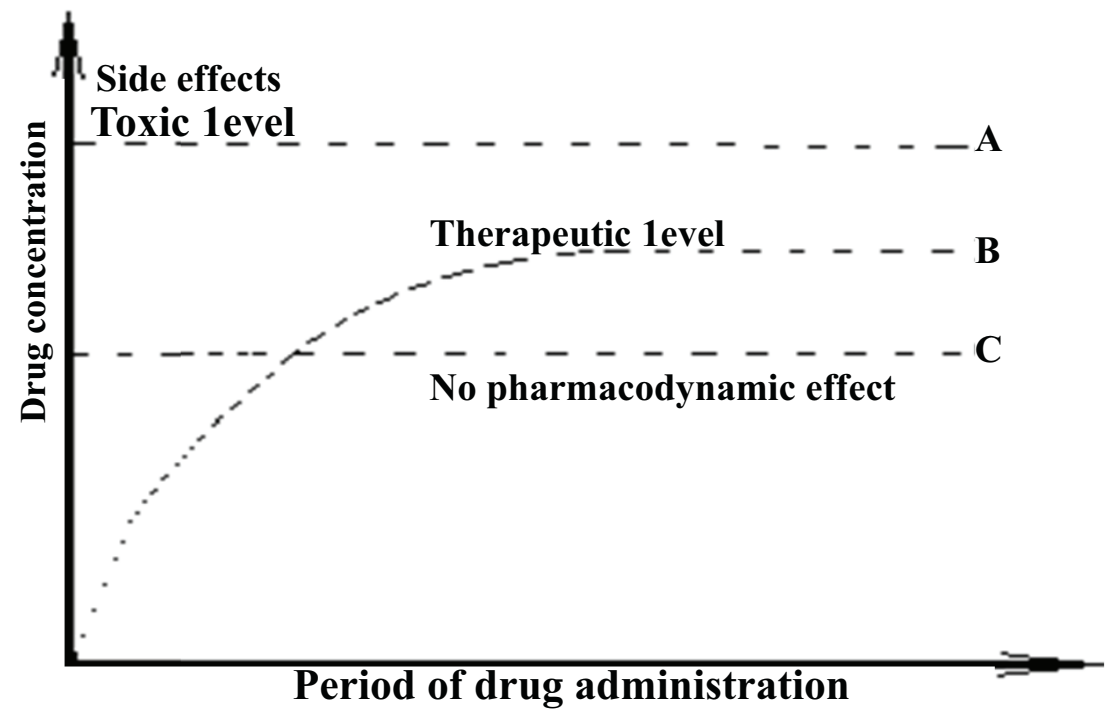

Fig. (1). Optimum domain of the variation of the sanguine concentration.

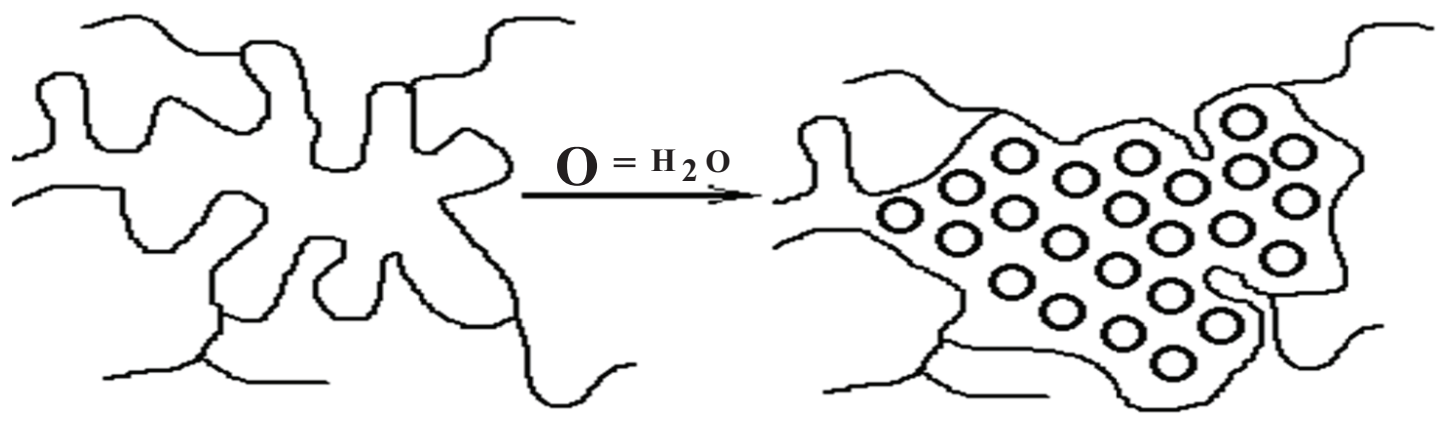

Fig. (2). Modification of the hydrogel polymer alignment after swelling in water.

certification of the realization of therapeutic concentration and the level corresponding to the diffusion speed can be carried out by means of the in vivo tests by monitoring the distance run by the drug coupled with a marker for co-focal microscopic visualization, for instance.

\subsection{Application of Drug-Containing Hydrogels on a Textile Surface}

The hydrogels are three dimensional organizations consisting of hydrophilic cross-linked polymers that form porous structures accessible to water molecules and biological fluids in significant amounts; the water molecules are fixed through hydrogen bonds to the functional groups of the hydrophilic polymer [3]. As a result of water fixation process, swelling effect occurs without the polymer dissolution under the influence of the physiological medium, which modifies the initial alignment of the polymer (Fig. 2).

The amplification of the swelling effect depends on the number of hydrophilic groups from the polymer chain, the cross-linking nature and frequency and the external stimuli; in the case of cutaneous pathologies, the $\mathrm{pH}$ value and the amount of perspiration, thermal effect of body temperature and the action of the enzymes from the skin surface also intervene. The hydrogels are biocompatible due to water retention.
The chemical synthesis pursues the production of some hydrogels in aqueous solutions with imposed properties, and the control of microstructure accuracy. From the textile point of view, what is of interest after the water extraction from the drug-containing hydrogel is the type of the obtained three dimensional network. After drying, the hydrogels form structures that shrink and preserve their initial shape, as well as discontinuous structures that after shrinking separate the surfaces, wrapping the continuous networks of the elementary volumes. The physical cross-linking has applicative potential, since it avoids the utilization of toxic cross-linking agents and does not need extraction operations.

The hydrogels are adequate to release drugs with big molecular mass, fragile, or susceptible to distortions or degradation during the usual processing. The drug release from hydrogels is performed through diffusion at molecular level, through the loops of the three dimensional network or through pores.

The hydrogels are used as surgical sutures, artificial organs, soft tissue prosthesis, hemodialysis membranes and contact lenses [3]. In order to realize a textile support with controlled release of a drug dispersed into the hydrogel, the following operations are necessary: 1. Hydrogel production; 2. Drug dispersion in hydrogel; 3. Application of drugcontaining hydrogel on the textile surface; 4. Manufacturing 


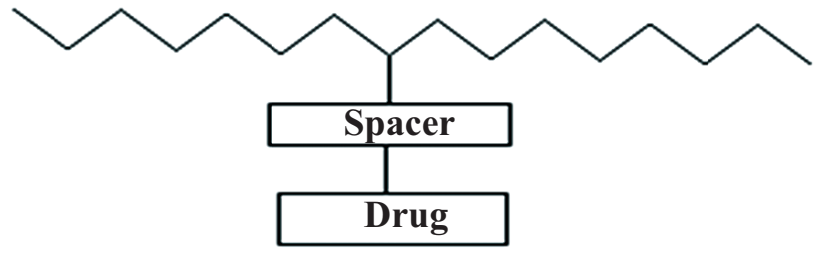

Fig. (3). Structure of a covalent polymer-drug conjugate.

the clothing ensemble; and 5. Clinical study on healthy volunteers and on patients with cutaneous pathologies.

\subsection{Matrix Systems with Drug Included for Controlled Release to the Patient Dermis}

Matrices are three dimensional networks that include the drug, the solvent and the excipients. The matrices use means inserting a drug in a hydrophobic matrix to accelerate the drug release speed or in a hydrophilic matrix to reduce the drug release speed, depending on the therapeutic target [4]. The matrix systems release antimicrobial and antifungal agents, as well as paclitaxel, a drug that prevents the neoplasm tissue to growth again after surgical excision. For hydrophobic matrices, one can use: ethyl-cellulose, polypropylene, polyethylene, wax, while for hydrophilic matrices one can use poly vinyl alcohol, acrylates and polyglycolic acid. There are inorganic porous matrices that have the advantage of biologic stability and releasing properties according to the pore dimension, topology and interaction of the pore surface with the drug, represented by zeolites, porous ceramics and silica.

The matrix systems consist of a suspended chain to which the drug is grafted on a macromolecule through covalent bonds by means of a spacer meant to sterically favor the reaction development. These are known as covalent polymer-drug conjugated, a name given by Ringsdorf and mean synthetic polymer drugs or pharmacologically active polymers. Ideally, the controlled release systems should determine the drug diffusion to the specific receiver at a specific duration and according to a specific release scheme, following the circadian rhythm, using polymers open to stimuli or intelligent polymers.

The advantages of polymer utilizations are due to: designing and preparation of some dosing forms with specific properties due to the polymer-polymer and polymerdrug interactions; the control of the release of some very toxic drugs or with severe adverse effects [4]; improvement of drug directing to specific receptors from tissues or cells; gathering the hydrophobic and hydrophilic portions within an individual structure. Fig. (3), adapted after the Ringsdorf model, presents the basic scheme of a drug grafted on a linear polymer structure.

In Fig. (3), the polymer can be natural or synthetic, and the spacer is obtained from a bi-functional reactive with the role to remove the drug from the polymer in order to intensify its reactivity in subsequent hydrolysis processes or enzyme attack inside a physiologic medium. The bond between the polymer chain and spacer is biodegradable, being of ester, amide, carbonate, anhydride or urethane nature. The matrices have as criteria: lack of toxicity; lack of mutagenicity; biodegradability; and sterilization capacity.

Development of polymer matrices with drugs as forms of dosage performs treatments with applications of multiple directions for therapies with smaller toxicity indices. Afterwards, the drug is split hydrolytically or with enzymes with a releasing kinetics due to splitting speed and diffusion of drug through the polymer network. The drug release from polymer matrices in solid form depends on a complex system of factors that involves simultaneous modifications of polymer and drug deep structure. Accordingly, the matrix swelling and erosion, drug dissolution (re-crystallization) and diffusion, matrix-drug structural interactions, drug distribution and concentration inside the matrix [5] as well as the matrix geometry, all represent from a qualitative standpoint, part of involved processes. The procedure could solve medical requirements for appropriate drug release by chemical syntheses but needs complex efforts for high accuracy.

\subsection{Drug Complexing with Cyclodextrin Fixed on Textile Surface}

The Cycs are cyclical oligosaccharides with abilities to encapsulate a series of guest molecules: compounds with polar behavior (amines, acids, esters, etc), aliphatic and aromatic hydrocarbons, rare gases, oxygen, carbon dioxide and polymers; they are obtained through enzyme conversion of Bacillus macerans starch. Cyclical glucose oligomers are obtained with 6 to 8 glucose units, named alpha-, beta- or gamma-Cyc having 1, 4-alpha-glycosidic bonds [6]. Cyc presents a good solubility in water (145-233 g/l) except for beta-Cyc that has a minimum solubility $(18.5 \mathrm{~g} / \mathrm{l})$.

The use of the capacity to complex the drug inside the internal cavity of Cyc modifies the drug dispersivity or solubility, or else its bio-accessibility inside the organism. The absorption of the hydrophobic drug inside Cyc determines the formation of an inclusion compound (IC). The oxyhydric groups external to Cyc solubilize the IC in the physiologic liquid. From a textile standpoint, the Cyc utilization induces a crisp handle in the cloth after the thermal cure necessary to fix the Cyc on the material. The above mentioned aspect can be solved punctually, but the procedure complicates the product realization. The existence of the temporary reservoir with the drug continuously released under the action of the dermal stimuli excludes an error in active principle administration, as happens in the conventional administration, dependent on the patient decision and correctness.

Here are the advantages of Cyc utilization: accessibility and capacity of natural regeneration of starch; simple ecofriendly manufacturing at an acceptable cost; possibility to synthesize some non-toxic Cyc derivatives. The IC formation needs a concordance between the molecule size and the size of Cyc hydrophobic cavity, for which there are softwares for dimensional evaluation of the guest drug. As a result of complexing, the whole hydrophobic molecule is dissolved in water. Higher solubility in physiologic liquid implies better bioavailability and faster diffusion. Among the three types, the mostly used is beta-Cyc, some derivatives of which were also used in the studies carried out during the 
recent years [3], such as: methyl-beta-Cyc, hydroxyl-propylCyc [7] or monochlorotriazine-beta-Cyc (MCT-beta-Cyc).

\subsection{Interaction of Cyclodextrin with Textile Support}

The Cyc can be deposited on the textile surface as an adhesive film. For the permanent fixation, the covalent grafting on cellulose is preferred. To make the application easier, one can use different reagents to link the Cyc on polymers as: acryloyl, epichlorohydrin or even the MCTbeta-Cyc; the last grafts cellulose under alkaline conditions.

The Cyc compounds are used for treatments with pharmaco-dynamic products, such as miconazole, with antimicotic action [6], flavonoids or corticosteroid derivatives as antiallergic therapy, or ciprofloxacin, as antibacterial released for a prolonged action inside the vascular prostheses, to diminish the risk of post-surgery infections [8]. A cross-linked polymer can be formed between the citric acid and Cyc, to increase the drug depositing capacity on a fibrous polymer, or to increase the period of drug release to the dermis; due to the polymer wettability, the compound can be created in a gel that also includes the drug, such that the release of the active principle provides a long-enough duration for a complete therapy.

The controlled-drug release determinations revealed the determinant role of Cyc, of the controlled-release medium, as well as of the guest. Starting from the idea to increase the drug releasing period, simultaneously with the realization of vascular prosthesis sealing, the Cyc crosslinking was performed with citric acid.

One can apply a multilayered coating on a surface as a useful instrument to increase the complexing capacity [9] of the fibrous polymer, with the amplification of controlled release durations.

\subsection{Trans-dermal Transportation of Drug from Microemulsions}

The microemulsions are mixtures consisting of water, oil, surfactant, and a co-surfactant in isotropic stable and clear form that perform the drug trans-dermal transport, improving its bio-availability [10]. They are useful in topical applications under various pharmaceutical forms, to be used as a drug carrier, applied on medical textiles, with an intensified skin penetration as compared to other procedures.

The microemulsions have an application potential, being studied as carrier at a lung level, for intra-rectal or vaginal administration for lipophilic drugs, as well as intramuscularly. In this case, the drugs are used for systemic therapies [10]. As penetration intensifiers, the saturated and unsaturated fat acids are used; the oleic acid, isopropyl palmitate, triglycerides with medium size chain, and other representatives. Surfactants are used biocompatible tensids: Tween $80^{\circledR}$, Tween ${ }^{\circledR}$ and others. The saturated alcohols: ethanol, propylene glycol and 1-butanol are used as cosurfactants.

The utilization of micro-emulsions is useful for drug trans-dermal transportation, as it is necessary in the case of psoriasis, since it increases the permeation through the corneous layer that represents the main diffusion barrier.

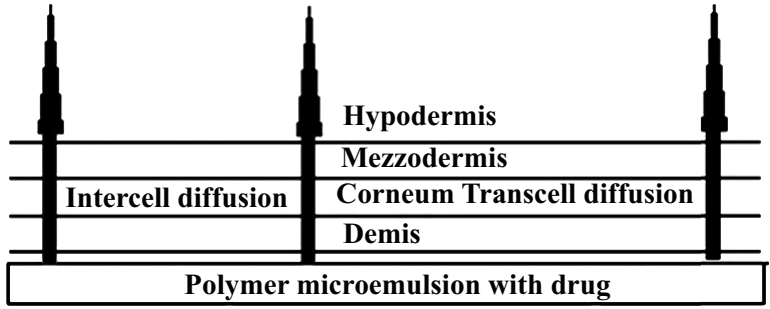

Fig. (4). Principle of drug diffusion from microemulsion to skin.

From an anatomic standpoint, the diffusion implies trans-cell and intra-cells paths, through sweat gland and the follicles of hairs located at dermis surface, in a differentiated extent depending on the skin location from the organism. For instance, the smallest permeation is at the heel area, which means a difficult transfer, but is also a location with abundant perspiration that favors the controlled release of Cyc. In Fig. (4), one illustrates the principle of drug transfer from microemulsion to human skin.

In order to obtain the textile material with drug meant to be released to dermis for cutaneous pathologies, the following stages must be run through: monitor the interest and the real need for a material with a self-contained drug or built-in a clothing fabric; choose the therapeutically potent drug; create the drug deposit on the textile surface; study the drug release; produce the clothing ensemble; clinical study on patients and healthy volunteers.

\subsection{Monitor the Interest and Advantage of Producing a Drug-Including Textile Material}

The study analyzes the realization of a textile medical support therapeutically more efficient than the existing medical procedures. Following the clinical considerations and the possibilities of textile processing, the criteria that need to be satisfied by the material as drug are sketched, as follows: form of presentation, the manner of assembling and wearing by the patient. It needs to be established if the medical textile support is to be used directly as therapeutic instrument shaped as stripes, bandages or fasciae, or it has to be included in a clothing ensemble as a blouse, trousers, under vest, etc. What concerns the destination, one could decide to produce either a disposable textile material, or a material that releases the drug during a time period, i.e. a releasing cycle, then washing it and possibly reloading with drug or wearing without reloading.

\subsection{Choose the Therapeutically Potent Drug}

The pharmacologist or the clinician establishes the drug specific to the dermis therapy. One estimates if its action is prophylactic or it treats the manifestations of a skin disease after affection outburst. One analyzes the necessity of a systemic treatment through which the drug transdermally diffuses in the presence or absence of a keratolytic action, or the surface therapies are only necessary for cutaneous manifestations.

Depending on the drug nature, the presence or absence of hydrophilic groups, the molecular mass and toxicity characteristics are structurally evaluated. 
In the case when the drug possesses hydrophilic groups and solubility, one should exclude the application through the formation of an IC with Cyc. In the absence of the hydrophilic groups, the drug has a lipophilic behavior. If the dimensional characteristics of length, width or branch length permit their inclusion in the Cyc hydrophobic cavity (with a mean inside diameter of cavity being $6.2 \AA$ in the case of beta component), the controlled release is made from the IC formed by the drug and beta-Cyc. If the dimensions exceed $6.2 \AA$, depending on the value of height or branches, one analyzes the utilization of gamma-Cyc or the possibility to give up the Cyc utilization and to use another release system. For the trans-dermal drug transfer, it is necessary to use a microemulsion that intensifies the diffusion through the corneous layer. When the drug is only applied on epidermis, the utilization of micro-emulsion is no longer necessary, being able to make a compound with Cyc, or formation of a hydrogel, depending on the punctual conditions of the drugfibrous polymer compound. For the use of a hydrogel the most sensible aspect is to avoid the burst effect of initial drug release. In the case of higher toxicity, one chooses to form a polymer matrix in which the drug is grafted to polymer. The drug has functional groups meant to be grafted to a polymer by means of a spacer.

\subsection{Create the Drug Deposit on the Textile Surface}

Practically, the quantity of drug applied on the textile material is calculated depending on the value DL50 of the drug, obtained through a toxicity study, as the value in $\mathrm{mg} / \mathrm{kg}$ body multiplied with the patient weight.

The therapeutic duration necessary for diffusion is assessed depending on the textile material type and the manner to produce the temporary drug reservoir and medical criteria. The realization of drug deposits is as well necessary when using matrix systems, the hydrogels or microemulsions. For a long-term diffusion that provides also a washing stage under spared conditions, the initial drug quantity provides the diffusion to the dermis for the entire therapeutic period. This implies the evaluation under detersive and turbulent conditions of the amount of drug lost as the effect of washing. That is why in the case when Cyc is used, the size of the Cyc quantity on the surface of the textile material is still limited by the possibility of a crisp handle.

In the case when the Cyc film physically deposed as a thin layer or grafted to the fibrous polymer chain provides the therapeutically necessary drug amount, but the drug has a high diffusion rate, it is necessary to create a cross-linking net outside the drug reservoir, to increase the space to be run by the drug to the dermis. On the contrary, when the diffusion rate determined in vitro is small, the drug quantity is amplified and the conditions that favor the diffusion must be determined. This case restricts the drug transfer rate from the textile material to dermis.

The work proposes the analysis of the possibilities to produce a textile medical article for a therapy specific to cutaneous pathologies, through the controlled release of a drug from a textile surface to dermis.

\section{MATERIALS AND METHODS}

One has used the $100 \%$ cotton interlock knitting as basic textile support for manufacturing a blouse and trousers, as well as a textile material treated with active principles or drugs [11].

The utilized reagents are: $\mathrm{FeCl}_{3}, \mathrm{~K}_{3} \mathrm{Fe}(\mathrm{CN})_{6}$, in commercial state, without purification treatments; an anionic surfactant (Bezema) and MCT-beta-Cyc (Wacker Chemie). The hydrocortisone acetate $(\mathrm{HCr})$ was obtained by courtesy of SC Antibiotice SA. For the application on the textile support one used: hydrocortisone acetate $(\mathrm{HCr})$; alcoholic extract (25\%) of Viola Tricoloris Herba (Vita) (a plant from spontaneous flora); propolis (Pro) harvested in 2010 from bees' families from Romania. Pro is conditioned, weighed and dissolved in ethylic alcohol (98\%) and filtered on filter paper. The filtrate is concentrated through evaporation, after which it is dissolved again in ethylic alcohol as a $30 \%(\mathrm{w} / \mathrm{v})$ solution; Menthol (Mtl) is found in Mentha piperita, but the extraction does not have a satisfying yield. That is why it was preferred to use the solid synthetic product (Sigma Aldrich) dissolved in $30 \%$ ethylic alcohol. In order to determine the dimensions of the active compounds molecules, we used the MarvinSpace ChemAxon 5.4.0.0 software.

Biological and toxicological studies were carried out for Vita and Mtl on mice and Guinea pigs with body mass of about 300-360 g. The performed procedure and experiments are used in pharmacology to determine the mean lethal dose of a product (noted by $\mathrm{DL}_{50}$ according to OECD 425 standards) and then to establish the therapeutic dose.

The tests for $\mathrm{HCr}$ controlled release from the cotton samples grafted with MCT-beta-Cyc and complexed were performed immersing them in the perspiration kit solution (prepared according to BS EN 1811-1999 standards, at a pH of 6.5 , that contains: $\mathrm{NaCl}$, lactic acid, urea and distilled water). The $\mathrm{HCr}$ solution in alcohol is colorless, the same as the carboxylic acid derived from $\mathrm{HCr}$ obtained through oxidation; one adds to the solution $\mathrm{FeCl}_{3}$ that is reduced to $\mathrm{FeCl}_{2}$ due to $\mathrm{HCr}$ oxidation, forming a colored ferrous salt [9]. In order to intensify the color with a view to examine the samples at UV/VIS spectra at $780 \mathrm{~nm}$, one adds potassium hexacyanoferrate to obtain blue-greenish hues, according to the reaction:

$$
\mathrm{K}_{3} \mathrm{Fe}(\mathrm{CN})_{6}+\mathrm{FeCl}_{2} \rightarrow \mathrm{KFe}\left[\mathrm{Fe}(\mathrm{CN})_{6}\right]+2 \mathrm{KCl}
$$

\section{RESULTS}

\subsection{Choose the Textile Support, the Drug and the Cyclodextrin}

The $100 \%$ cotton knitting was chosen as a textile support destined to manufacture a blouse and trousers for patients with atopic dermatitis and contact dermatitis. The interlock structure is usual for personal underwear (night clothes) garments. The choice of cotton cellulose was preferred due to the good reactivity of the $\mathrm{OH}$ groups, as well as to the possibility to obtain softness, wettability and a good 


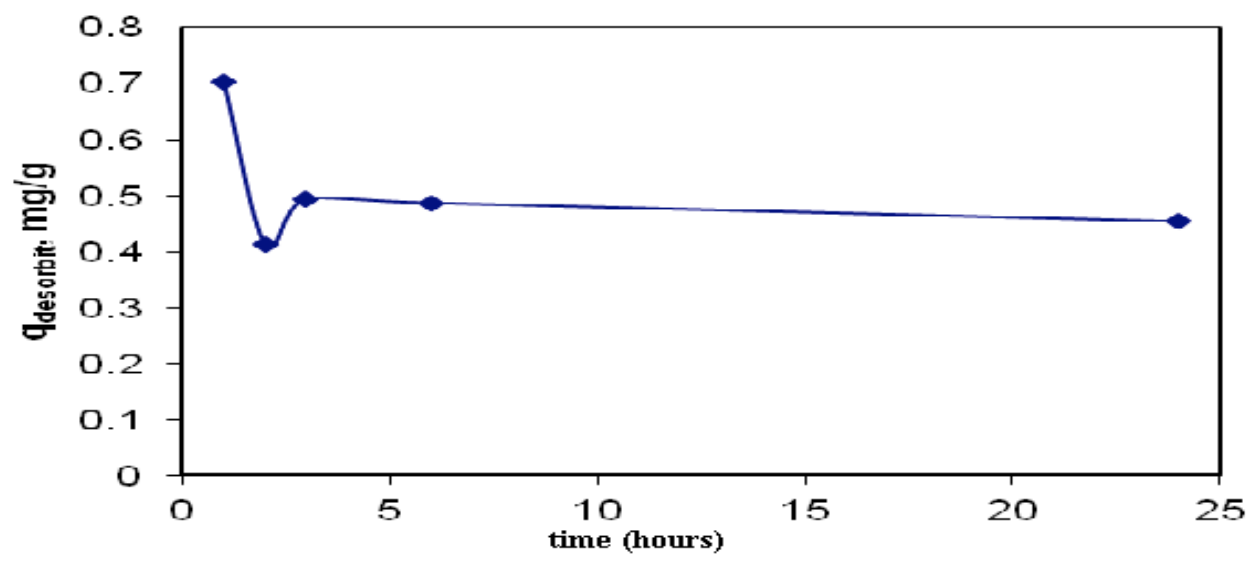

Fig. (5). Desorption curve of HCr from textile to same solution of perspiration kit at different times.

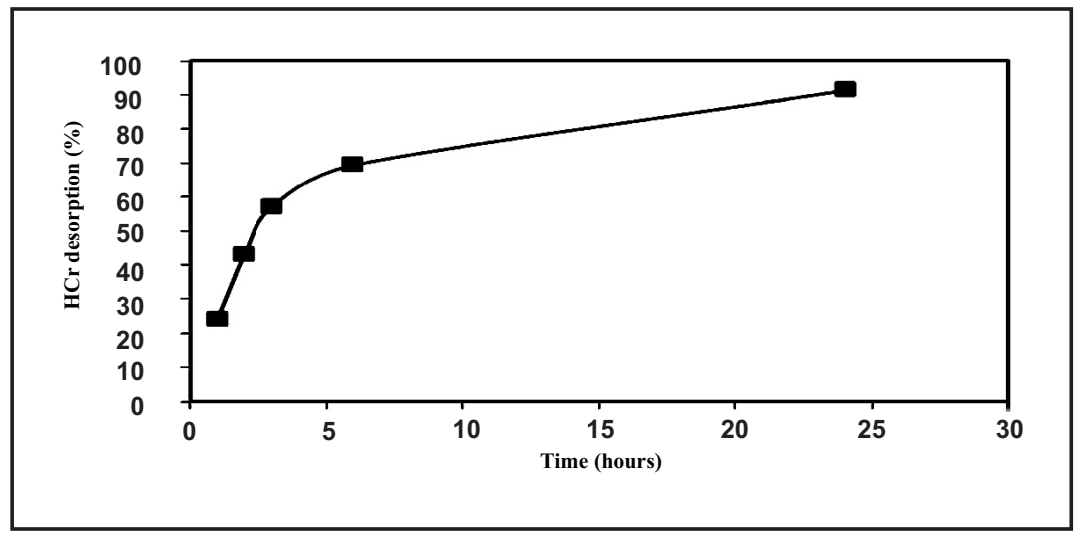

Fig. (6). Desorption curve of $\mathrm{HCr}$ from textile to perspiration kit after immersion, desorption and drying.

sterilization capacity. Cellulose has a chemical reactivity favorable to grafting.

\subsection{Preparation of Textile Support}

The cotton knitting follows the next stages: 1. Alkaline boiling then hot and cold washing, to obtain a hydrophilic material; 2. Bleaching with hydrogen peroxide for a good sorption capacity and sterilization; 3. Warm rinsing; 4. Drying: the interlock knitting antiallergically treated is subjected to the following additional operations; 5. Grafting with MCT-beta-Cyc; 6. Spraying the active principle on the surface of the textile material; fixation at $50^{\circ} \mathrm{C}, 4 \mathrm{~h}$; 7. Manufacturing the textile material. The compound formed between cellulose and MCT-beta-Cyc absorbs inside the cyclodextrin cavity in each of the active principles (hydrophobic products). The nocturnal perspiration determines the release of the active principle from the textile surface to the dermis of the patient with dermatitis. This results in the therapeutic effect of the utilized active principle. The cellulose support favorably responds to the requirement of forming a reservoir of antiallergic drug simultaneously with getting an increased cutaneous comfort.

\subsection{Evaluation of Bioactive Compounds Dimensions}

The Table 1 illustrates the dimensions of the compounds from the active principles, obtained by using the MarvinSpace ChemAxon 5.4.0.0.

\subsection{In Vivo Tests}

As the result of the toxicity tests, the following experimental values for the lethal doses were obtained: $\mathrm{DL}_{50}$ $\mathrm{Mtl}=500 \mathrm{mg} / \mathrm{kg}$ body, DL50 Vita= $200 \mathrm{mg} / \mathrm{kg}$ body, and for Pro the value DL50 Pro $=400 \mathrm{mg} / \mathrm{kg}$ body, in accordance with the previous literature [12]. For some products, antioxidant effect was obtained by testing the level of enzyme superoxide dismutase. For testing, lots of Guinea pigs with weights from 300 up to $360 \mathrm{~g}$ were used. The results were compared with values obtained using a saline solution. The highest value was recorded in case of propolis alcoholic solution administered parenterally.

\subsection{Determination of $\mathrm{HCr}$ Controlled Release}

Figs. (5 and 6) illustrate the quantity of $\mathrm{HCr}$ released by the material with MCT-beta-Cyc immersed in the solution of perspiration kit. The cotton was chosen due to the good reactivity of the $\mathrm{OH}$ groups from cellulose, as well as to the possibility to obtain softness, wettability and a good sterilization capacity. The details on textile performances have been communicated elsewhere.

\section{DISCUSSION}

The compound formed between cellulose and MCT-betaCyc absorbs in Cyc cavity in each of the active principles or hydrophobic pharmaceutical products. After manufacturing 
Table 1. Dimensional values of the drug/active principles.

\begin{tabular}{|c|c|}
\hline Compound & Dimensions $(\mathbf{A})$ \\
\hline \hline Beta-Cyc & 1. Height=7.8; 2. Inner diameter $=6.2$ \\
\hline HCr & $1.12 .21 ; 2.5 .69 ;$ antiallergic effect \\
\hline Methyl prednisolone & $\begin{array}{c}\text { Anti-inflammatory effect } \\
1.12 .485 ; 2.5 .644\end{array}$ \\
\hline Ferulic Acid & $\begin{array}{c}\text { A flavonoid component from Vita and Pro. } \\
1=8.77 ; 2=4.46 ; 3=4.46 ; \text { anti-allergic }\end{array}$ \\
\hline Flavonoid & $\begin{array}{c}\text { Main components from Vita and Pro. } \\
1=10.57 ; 2=6.39 ; 3=4.01 ; \text { anti-allergic }\end{array}$ \\
\hline Menthol & $\begin{array}{c}\text { Cooling effect on dermis. } \\
1=6.94 ; 2=4.28 ; 3=2.54\end{array}$ \\
\hline Pimecrolimus & \begin{tabular}{c}
$1=14.783 ; 2=14.656 ;$ psoriazis therapy \\
\hline
\end{tabular}
\end{tabular}

Legend: 1. Length; 2. Maxim width; 3. Current width.

the textile articles, the nocturnal transpiration determines the release of the active principle from the textile surface to the dermis of the patient with dermatitis; this results in the therapeutic effect of the used active principle. The cellulose support favorably responds to the requirement to create a reservoir of antiallergic drug, simultaneously with getting an increased skin comfort.

Vita and Pro contain anthocyanins, ferulic acid, solanine and flavonoids. By comparing the maximum width of the compounds from Table 1, with the inner diameter of betaCyc, one can notice that the flavonoids, ferrulic acid, menthol, and methyl prednisolone illustrated in Fig. (1) can be adsorbed inside the Cyc. On the other side, $\mathrm{HCr}$ and hydrocortisone butyrate are dimensionally adequate for Cyc inside. Obviously, the drugs with the width value higher than the inner diameter of Cyc cannot form an inclusion complex.
The tests of controlled release of flavonoids (unpublished), as well as their efficacy, have revealed the therapeutic value of the extract of Vita and Pro.

For drug dosing on the textile material and for antiallergic tests the following therapeutic doses (t.d.) were used: t.d. $\mathrm{Mtl}=8.203 \mathrm{mg} / \mathrm{kg}$ body; t.d. VITA= 4.53125 $\mathrm{mg} / \mathrm{kg}$ body and t.d. Pro $=10 \mathrm{mg} / \mathrm{kg}$ body, correlated with the DL50 values. For a blouse and trousers destined to a person of $60 \mathrm{~kg}$ for instance, the amount of Vita applied on the knitting grafted with MCT-beta-Cyc is calculated with the relation: Quantity of Vita $=60 \times$ t. d.Vita $=60 \times 4.53215=$ $271.875 \mathrm{mg}$ pure product. Afterwards, the material treated with active principles (Vita, Mtl, Pro) is assembled customized inside the clothing, depending on the location of the dermis eruption. The alcoholic solution for Pro and Mtl application is calculated similarly.

Nearby well-known anti-allergic effect Pro has an antioxidant enzyme SOD of 1500 units/ml important for a patient.

The tests of $\mathrm{HCr}$ release illustrated in (Figs. 5 and 6) show that, irrespective of the working method, there is an equilibrium between the quantity of $\mathrm{HCr}$ complexed in $\mathrm{Cyc}$ and the one released through perspiration. Replacing the kit that already has absorbed $\mathrm{HCr}$ with a new amount of kit shifts the balance towards $\mathrm{HCr}$ desorption and the diffusion to dermis, but the process does not follow the profile of Langmuir law. A scheme of diffusion process is illustrated in Fig. (7).

It can be noticed from Fig. (7) that the medicine drug is set inside Cyc and on cellulose hydroxylic groups as hydrogen linkage. For an accurate control of drug release from textile surface, a procedure able to have an interaction of drug only with Cyc has to to be promoted.

In the sense of the studied pathology, the cutaneous physiology is complex. The tests performed in the Laboratory of I.A.T.T. from Citta degli Studi di Biella (Italy) indicate the following: the trans-dermal water loss, water

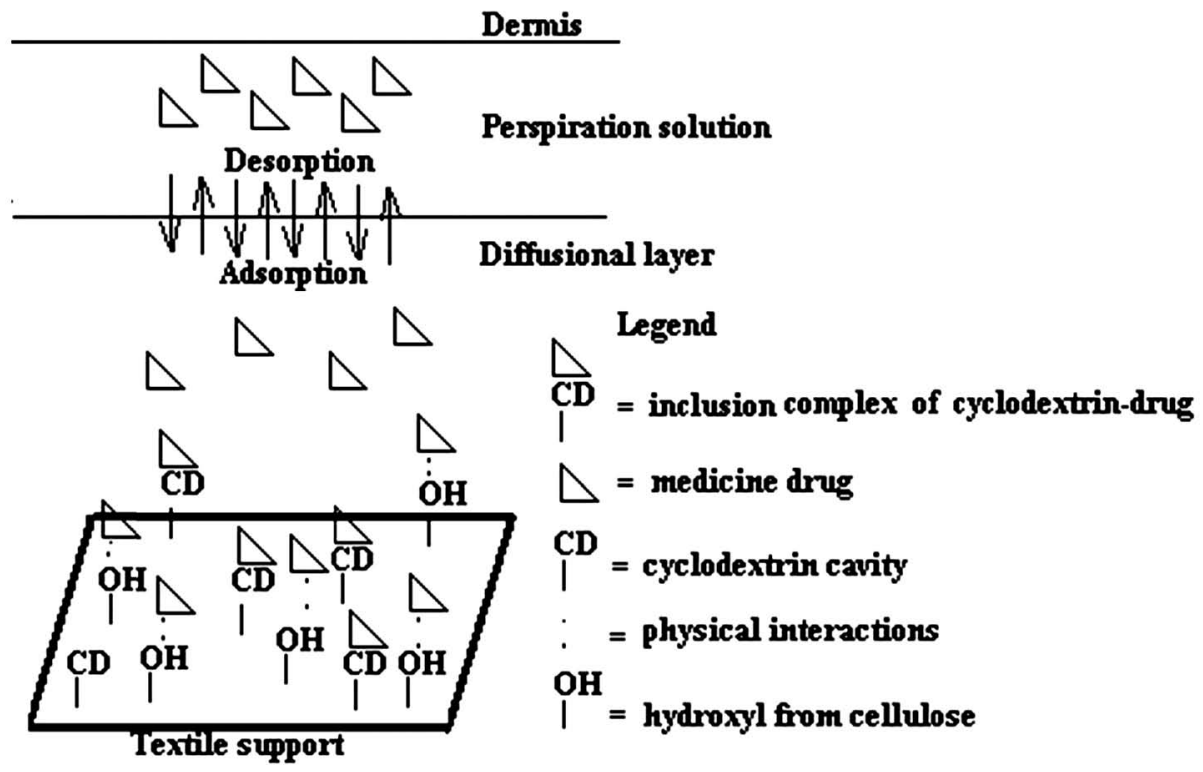

Fig. (7). Diffusion principle of medicine drug both from textile and IC to dermis. 
loss through the total skin surface, hydration level, irritation level, dermis $\mathrm{pH}$ and the blood circulation were performed on the knitting with and without active principles. The analysis of the physiological data obtained on volunteers on the forearm zone led to the conclusion that the treatments carried out on the knitting did not induce an aggravation of the dermis physiology.

After confectioning the blouse and trousers the knitting pieces treated with MCT-beta-Cyc, Vita, Mtl or Pro are fixed at the inside part. The zone covered with antiallergic treated knitting is indicated by the clinician, depending on the dermatitis type, the patient anthropometric dimensions and the specific of pathology.

Generally, the clothes for patients with atopic and contact dermatitis are designed according with specific dimensions of each patient. What concerns the diffusion rate, the controlled release of the active principles under the action of cutaneous perspiration must follow the medical prescriptions and provide a controlled release for a period of time corresponding to the capacity of knitting loading with active principles between the two therapeutic concentrations: minimum and maximum (see Fig. (1) between $\mathrm{A}$ and $\mathrm{C}$ limits). The realization of the therapeutic dose represents a decisive factor. The details of the involved processes are also associated with the conclusions of the clinical tests under the conditions given by the peculiarities of each patient and by cutaneous manifestation.

\section{CONCLUSION}

The cutaneous affections that can be approached through therapies of drug release from a textile support have an applicative potential, even if they represent only a small niche. The application of matrix systems, microemulsions or hydrogels can overcome the deficiencies and shortcomings due to Cyc utilization. In the case of inclusion compound between $\mathrm{HCr}$ and beta-Cyc, additional experiments are necessary to obtain a profile of controlled release within the therapeutic limits. The utilization of MCT-beta-Cyc as support for the generation of an IC with active principles used in this work is a favorable solution which has to be adjusted according with drug behavior to obtain a convenient controlled release system. The presented stages of textile support processing can also be extended to the application of some pharmacologic antiallergic products, depending on: the performances determined in vivo; molecular dimensions of the drug that need to penetrate inside the cyclodextrin cavity.
Cotton knitted fabric represents a promising medical support for chemical modification in some drug release applications.

\section{CONFLICT OF INTEREST}

The authors confirm that this article content has no conflict of interest.

\section{ACKNOWLEDGEMENTS}

The research is funded by PN II-72 148 project.

\section{REFERENCES}

[1] D'Antuono, A.; Baldi, E.; Bellavista, S.; Banzola, N.; Zauli, S ; Patrizi, A. Use of Dermasilk briefs in recurrent vulvo-vaginal candidosis: safety and effectiveness. Mycoses, 2012, 55(3), 85-89.

[2] Masajtis, J.; Dutkiewicz, J.; Członka, R.; Broniarczyk-Dyła, G.; Arkuszewska, C.; Skwarczyńska-Bany, E.; Omulecki, A.; Kot, P. Correlations between the properties and effects of clinical therapy using textile material with antipsoriatic action. Polym. Med., 1992, 22(1-2), 26-41.

[3] Wang, C.; Stewart, R. J.; Kopecek, J. In: Polymeric Drugs \& Drug Delivery Systems, Ottenbrite, K., Ed.; CRC Press: Boca Raton, 2000, pp. 131-140

[4] Grassi, M.; Grassi, G.; Lapasin,R.; Colombo, I. Understanding Drug Release and Absorption Mechanisms. A Physical and Mathematical Approach; CRC Press, New York, 2006.

[5] Grassi, G.; Hasa, D.; Voinovich, D.; Perissutti, B.; Dapas, B.; Farra, R.; Franceschinis, E.; Grassi. M. Simultaneous release and adme processes of poorly water-soluble drugs: mathematical modeling. Mol. Pharm., 2010, 8, 1488-1497.

[6] Wang, JH.; Cai, Z. Investigation of inclusion complex of miconazole nitrate with beta-ciclodextrin. Carb.Polym., 2008, 72, 255-260.

[7] Freitas, M.R.; Rolim, L.A.; Roca Soares, M.F.; Rolim-Netob, P.J.; Albuquerquea, M.M.; Soares-Sobrinho, J.M. Inclusion complex of methyl-cyclodextrin and olanzapine as potential drug delivery system for Schizophrenia, Carb.Polym., 2012, 89, 1095-1100.

[8] Blanchemain, N.; Karrout, Y.; Tabary, N.; Bria, M.; Neut, C. Hildebrand, H.F.; Siepmann, J.; Martel, B. Comparative study of vascular prostheses coated with polycyclodextrins for controlled ciprofloxacin release. Carb.Polym., 2012, 90, 1695-1703.

[9] Martin, A.; Tabary, N.; Leclercq, L. Multilayered textile coating based on a beta-cyclodextrin polyelectrolyte for the controlled release of drugs. Carb. Polym., 2013, 93, 718-730.

[10] Kogan, A.; Garti, N. Microemulsions as transdermal drug delivery vehicles. Adv. Coll. Interf. Sci., 2006, 123-126, 369-385.

[11] Radu, C. D.; Salariu, M.; Avadanei, M.; Ghiciuc, Cr.; Foia, L.; Lupusoru, E. C.; Ferri, A.; Ulea, E.; Lipsa. Fl. Cotton-made cellulose support for anti-allergic pajamas. Carb. Polym., 2013, 95, 479-486.

[12] Burdock, G.A. Review of the biological properties and toxicity of bee propolis (Propolis); Food Chem. Toxic., 1998, 347-363. 